\title{
Optimisation of an asymmetric polymerase chain reaction assay for the amplification of single-stranded DNA from Wuchereria bancrofti for electrochemical detection
}

\author{
Vasuki Venkatesan ${ }^{1 /+}$, Sugeerappa Laxmanappa Hoti ${ }^{1}$, \\ Nagalakshmi Kamaraj', Somnath Ghosh'², Kaushik Rajaram² \\ ${ }^{1}$ Vector Control Research Centre, Indira Nagar Medical Complex, Pondicherry, India ${ }^{2}$ Indian Institute of Science, Bangalore, India
}

Single-stranded DNA (ssDNA) is a prerequisite for electrochemical sensor-based detection of parasite DNA and other diagnostic applications. To achieve this detection, an asymmetric polymerase chain reaction method was optimised. This method facilitates amplification of ssDNA from the human lymphatic filarial parasite Wuchereria bancrofti. This procedure produced ssDNA fragments of $188 \mathrm{bp}$ in a single step when primer pairs (forward and reverse) were used at a 100:1 molar ratio in the presence of double-stranded template DNA. The ssDNA thus produced was suitable for immobilisation as probe onto the surface of an Indium tin oxide electrode and hybridisation in a system for sequence-specific electrochemical detection of $\mathrm{W}$. bancrofti. The hybridisation of the ssDNA probe and target ssDNA led to considerable decreases in both the anodic and the cathodic currents of the system's redox couple compared with the unhybridised DNA and could be detected via cyclic voltammetry. This method is reproducible and avoids many of the difficulties encountered by conventional methods of filarial parasite DNA detection; thus, it has potential in xenomonitoring.

Key words: asymmetric PCR - single-stranded DNA - electrochemical detection - Wuchereria bancrofti

Lymphatic filariasis (LF), one of the most important neglected tropical diseases, continues to batter the social and economic wellbeing of people in underdeveloped/ developing nations. LF is caused by the lymphatic system-dwelling nematode parasites Wuchereria bancrofti, Brugia malayi and Brugia timori and is transmitted by vector mosquitoes. It has been targeted for an elimination programme that is largely based on repeated annual cycles of mass drug administration (MDA) using an antifilarial drug against endemic populations (Ottesan 2000). New diagnostic tools and strategies are essential at every stage of monitoring and evaluation during the elimination programme (WHO 1997).

The recent introduction and development of molecular technologies have moved filarial parasite-detection systems from conventional methods of dissection and microscopy (which are labour-intensive, tedious and often impractical) to improved polymerase chain reaction (PCR)-based assays that have considerable potential for field use (Farid et al. 2001). The SspI PCR assay has shown promise in detecting $W$. bancrofti infection in blood and mosquitoes in a number of laboratories through improved methods (Ramzy et al. 1997, Hoti et al. 2002, Helmy et al. 2004). However, improvement in the processing efficiency of PCR-based assays in terms of cost and time becomes imperative for routine monitor-

doi: 10.1590/0074-0276108062013020

Financial support: DST

+ Corresponding author: vvasuki@yahoo.com

Received 15 November 2012

Accepted 18 December 2012 ing of infection in pools of vectors during the post-MDA period. Though existing PCR assays may be refined by adopting a simple, rapid and inexpensive method of DNA extraction from the filarial vector Culex quinquefasciatus infected with $W$. bancrofti (Vasuki et al. 2003), resolving the PCR product still relies on agarose gel electrophoresis followed by ethidium bromide (EtBr) staining to detect the parasite-specific DNA band based on its molecular size. This is a time-consuming, low-sensitivity and cumbersome process, apart from being hazardous to lab personnel due to the use of $\mathrm{EtBr}$ for staining the gel. Alternative methods such as real-time PCR (Vasuki et al. 2012) provide higher sensitivity, but are expensive and unwieldy. To overcome these problems, an electrochemical biosensor-based approach was attempted for the detection of filarial infection in vectors.

We designed an electrochemical DNA biosensor consisting of an electrode surface modified with an oligonucleotide probe complimentary to a target DNA analyte. In this system, when the target hybridises with the probe, the hybrid formation can be electrochemically transduced using a methylene blue (MB) indicator-based approach (Lai et al. 2006). For the detection of parasite infection using the electrochemical sensor, it is necessary to have single-stranded DNA (ssDNA) to be used as both the probe and the target. PCR amplification normally results in a double-stranded DNA (dsDNA) product. Use of a double-stranded linear PCR product is notoriously unreliable because of rapid reannealing of the denatured DNA (Li et al. 2004). Therefore, an asymmetric PCR technique was optimised to generate an excess of ssDNA for use in electrochemical detection. This enabled us to achieve the sensitivity and reproducibility of electrochemical detection and the results are presented here. 


\section{MATERIALS AND METHODS}

Microfilariae of $W$. bancrofti were isolated using a Percoll-gradient centrifugation technique from blood collected from a microfilaria carrier after obtaining his written informed consent as approved by the Ethical Committee of our Centre. Laboratory-reared $C x$. quinquefasciatus was obtained from insectaries maintained at our Centre.

Asymmetric PCR assay - Initially, asymmetric PCR was performed in two steps (Kaltenboeck \& Kousoulas 1996). In the first step, primary PCR was performed using a 25 reaction mixture containing $2.5 \mu \mathrm{L}$ of $10 \mathrm{x}$ PCR buffer, $1.0 \mu \mathrm{L}$ of $25 \mathrm{mM} \mathrm{MgCl}, 3.0 \mu \mathrm{L}$ of $10 \mathrm{mM}$ deoxynucleotide triphosphates, $1.5 \mu \mathrm{L}$ each of forward primer NV1, 5-CgtgATggCATCAAAgTAgCg-3' and reverse primer NV2, 5'-CCCTCACTTACCATAAgACAAC-3', with each primer at a $10 \mathrm{pM}$ concentration, $0.5 \mu \mathrm{L}$ of Taq polymerase enzyme, $5.0 \mu \mathrm{L}$ of template [ $W$. bancrofti genomic DNA extracted using the simple TE method (Vasuki et al. 2003)] and $10 \mu \mathrm{L}$ of water. The PCR followed a protocol of $95^{\circ} \mathrm{C}$ for $5 \mathrm{~min}$ and 35 cycles of $95^{\circ} \mathrm{C}$ for 1 $\min , 55^{\circ} \mathrm{C}$ for $1 \mathrm{~min}, 72^{\circ} \mathrm{C}$ for $1 \mathrm{~min}$ and a final extension at $72^{\circ} \mathrm{C}$ for $10 \mathrm{~min}$. The product obtained in the primary PCR was used as a template in the secondary PCR step with one of the primers (forward or reverse) in excess. To achieve successful amplification, further optimisation was attempted by varying the concentrations of the ingredients in the PCR reaction. Optimisation was also performed by adopting different time and temperature regimes and numbers of thermal cycles.

To generate an excess of ssDNA of both sense and anti-sense strands necessary for binding as probes to the Indium tin oxide (ITO) surface and to use as the target, respectively, we combined this optimised asymmetric PCR technique into a single-step assay. Products were generated using the amplification protocol as given above at a 100:1 molar ratio of the reverse:forward or forward:reverse primers. The single-stranded products were visualised using an ultraviolet transilluminator after electrophoresis on a $2 \%$ agarose gel in Tris-acetateethylenediamine tetraacetic acid buffer with $\mathrm{EtBr}$ stain. The $188 \mathrm{bp}$ amplified product bands were cut from the gel, purified (Nucleospin, Macherey Nagel) and quantified (GeneQuant, Amersham Biosciences), and their sequences were confirmed by sequencing (Applied Biosystems-3130XL, USA). The utility of the purified ssDNA generated in detecting $W$. bancrofti parasite DNA was verified by electrochemical detection using a prototypic electrochemical cell (E-cell)we developed and a cyclic voltammeter (CV).

A small Pyrex glass E-cell suitable for the detection of biological samples was fabricated in-house. The typical dimension of the cell was $3 \mathrm{~cm} \times 2 \mathrm{~cm}$ and the maximum volume that it could hold was approximately $7.0 \mathrm{~mL}$. The E-cell consisted of a $1 \mathrm{~cm} \mathrm{X} 1 \mathrm{~cm}$ ITO electrode, which served as the working electrode, a piece of platinum foil as the counter electrode and $\mathrm{Ag} / \mathrm{AgCl}$ as the reference electrode. The ITO working electrode served as the substrate for immobilisation of the ssDNA (Probe) (Fig. 1).
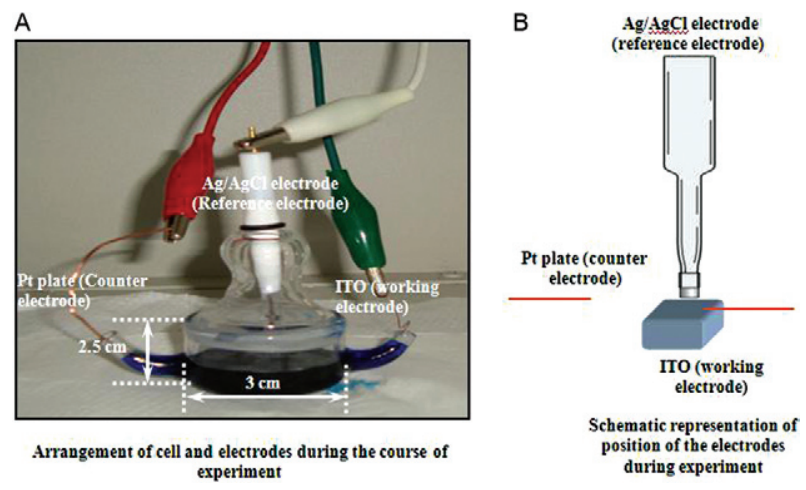

Fig. 1: electrochemical cell arrangement. A: arrangement of cell and electrodes during the course of experiment; B: schematic representation of position of the electrodes during experiment; ITO: Indium tin oxide; Pt: platinum.

To immobilise ssDNA onto the ITO electrode, the ITO surface was thoroughly cleaned with a surfactant followed by Millipore water and dried. The ITO surface was hydroxylated by dipping the electrode into $2 \mathrm{M} \mathrm{KOH}$ for $5 \mathrm{~h}$. It was silinated by dipping it into a $2 \%$ concentration of aminopropyl triethoxy silane (APTS) for an hour and was repeatedly washed with Millipore water to remove unattached APTS. The modified ITO was dipped for over a period of $24 \mathrm{~h}$ at $45^{\circ} \mathrm{C}$ into a known concentration of ssDNA. The $-\mathrm{NH}_{2}$ groups of the silane were attached to the 5 '- $\mathrm{PO}_{4}^{3-}$ groups of the ssDNA, forming a strong covalent bond. The modified electrode was used in the E-cell and a $\mathrm{CV}$ was recorded using an electrochemical workstation (Fig. 1) with a scan rate $50 \mathrm{mV} / \mathrm{sec}$ at a very low concentration of MB. The electrochemical workstation used was an electrochemical analyser (CH Instruments, model NO608C) when electrochemical techniques such as CV, Linera sweep voltammetry, Tafel plot, chronocoulommetry and chronoamperometry were performed. Our instrument is equipped with a picoampere booster and a Faraday cage to detect even a small response current. The electrode was lightly washed with Millipore water and immersed in a solution containing the target DNA for over a period of 16 $\mathrm{h}$ at $45^{\circ} \mathrm{C}$ for hybridisation. Finally, the $\mathrm{CV}$ was recorded at the same scan rate $(50 \mathrm{mV} / \mathrm{sec})$ with $\mathrm{MB}$, which intercalates into the major groove of DNA.

\section{RESULTS}

In a search for new tools and strategies for monitoring and evaluating filariasis for elimination campaigns, a nanotechnology-based approach was attempted for the sequence-specific electrochemical detection of $W$. bancrofti-specific PCR products. ssDNA is a prerequisite for the electrochemical sensor-based detection. Hence, optimisation of the amplification of a single-stranded $W$. bancrofti-specific 188 bp fragment of DNA by asymmetric PCR was performed to generate an excess of ssDNA fragments for use in electrochemical detection.

Asymmetric PCR was used to preferentially amplify the sense strand of the original DNA to a greater extent than the anti-sense strand. Asymmetric PCR is useful 


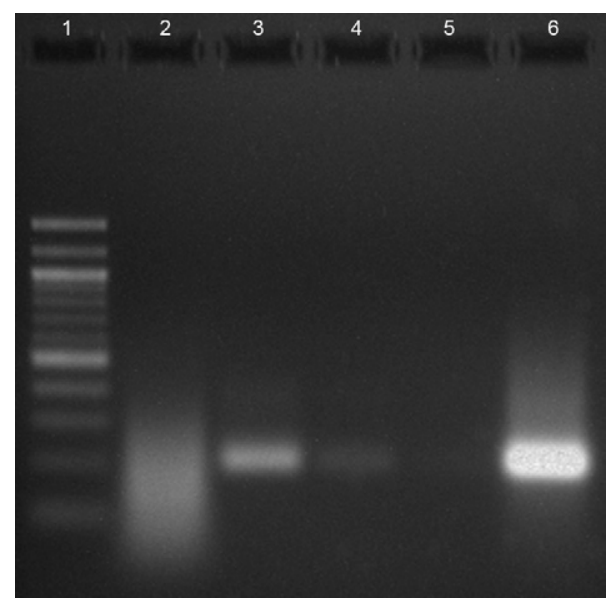

Fig. 2: electrophoresis patterns of symmetric and asymmetric polymerase chain reaction products in $2 \%$ agarose gel. Lane 1: DNA marker 100 bp; 2: negative control (100:1); 3-5: asymmetrically amplified ssDNA at 100:1, 50:1 and 10:1 (forward:reverse) primer molar ratio respectively; 5 : symmetrically amplified dsDNA using 1:1 primer molar ratio.

in hybridisation probing in which only one of the two complementary stands is required. The asymmetric PCR assay was initially performed using two steps: dsDNA amplified in the first reaction was used as the starting material for production of ssDNA in a second PCR with one primer in excess. Further optimisation to prevent nonspecific amplification and background bands resulted in the production of mainly the ssDNA sense strand in a single step with a 100:1 molar ratio of the forward and reverse primers and an extension time of $7 \mathrm{~min}$, which was useful for making ssDNA probes. Using the same 100:1 molar ratio of the reverse and forward primers, ssDNA of the antisense strand was amplified under optimised conditions for use as the template DNA.

\section{DISCUSSION}

We achieved minimisation of the time and the cost involved in the two-step reactions by optimising singlestep reactions by varying the primer concentrations to obtain good amplification (Fig. 2). Similarly, by optimising an asymmetric PCR, successful amplification of ssDNA was reported in the electrochemical detection of the 5S-rRNA spacer region of the traditional Chinese medicinal herb Fritillaria thunbergii (Li et al. 2004). Fluorescent data analysis demonstrated that a significantly higher level of fluorescence signal, hence higher detection sensitivity, was obtainable using asymmetric PCR than symmetric PCR in the presence of a molecular beacon probe (Poddar 2000). ssDNA molecules were generated by asymmetric PCR incorporating cross-linking analogues into specific sites in a DNA probe (Wooddell \& Burgess 1996) and were used for the detection of dengue virus RNA using an electrochemical biosensor (Rai et al. 2012). Carbon nanotubes (CNTs) tend to form bundles in solution, which is one of the barriers to their application. CNTs can be effectively dispersed and func-

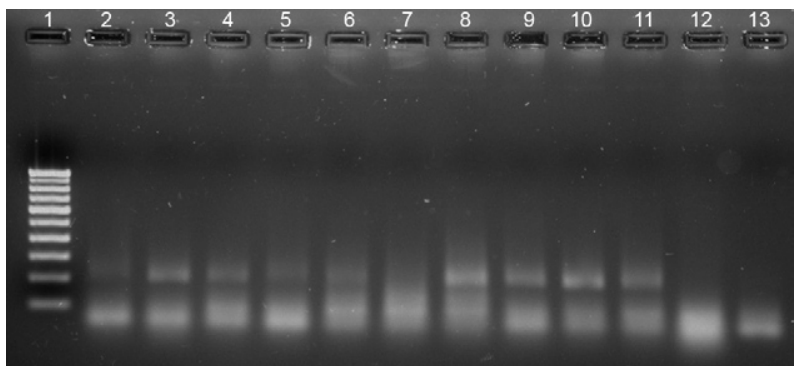

Fig. 3: amplification of single-stranded DNA in pooled mosquito vectors by asymmetric polymerase chain reaction with 100:1 molar ratio of primers. 1: DNA marker $100 \mathrm{bp}$; 2-6: sense strand; 7-10: antisense strand; 11: positive control; 12: negative control; 13: negative control.

tionalised by wrapping them with long ssDNA synthesised by asymmetric PCR to facilitate electron transfer reaction at an electrode surface in the development of novel electrochemical biosensors (Liang et al. 2007).

The production of ssDNA probes was performed initially after sufficient optimisation using pure genomic DNA from the parasite. Because our ultimate aim was to detect the parasite in vectors, production of ssDNA was standardised using samples containing single microfilaria in pools of mosquitoes. We standardised the assay using one mf per pool of 10 mosquitoes, which produced a positive signal when the resulting template was used at a 1:20 dilution. Further increasing the mosquito pool size from 10-25 did not produce any positive amplification signal using the same composition of PCR reagents and the standard amplification program.

To achieve consistent amplification in samples with pooled (10) mosquitoes, a ready-to-use master mix (Promega, USA) was used, with the composition of $12.5 \mu \mathrm{L}$ Master mix, $7.0 \mu \mathrm{L}$ of template (1:20 dilution) and $2 \mu \mathrm{L}$ of the required concentration of the forward/upstream primer and $2.0 \mu \mathrm{L}$ of the downstream/reverse primer made up to $25 \mu \mathrm{L}$ with nuclease-free water (Fig. 3). The amplified products were purified, quantified, sequenced and confirmed with the known sequence. This product was utilised for the electrochemical detection of the $W$. bancrofti parasite in the vectors.

The results of the CVs obtained at various stages using ITO in a phosphate buffer ( $\mathrm{pH}$ 7.2) are shown in Fig. 4. In the case of the hydroxylated and silinated ITO, no characteristic redox peak corresponding to MB was observed. However, a neat redox peak was observed in the presence of the ssDNA (probe) and dsDNA after hybridisation. There were considerable decreases in both the anodic and the cathodic currents of the redox couple in the case of the hybridised DNA compared with that of the unhybridised DNA. The amount of the free MB available in solution after hybridisation was less because a part of the MB had been intercalated into the dsDNA and was not available for the electrochemical reaction. Thus, one can use this type of E-probe conveniently for the qualitative detection of $W$. bancrofti DNA in samples.

In summary, PCR conditions were optimised for the synthesis of ssDNA by asymmetric PCR. This method 


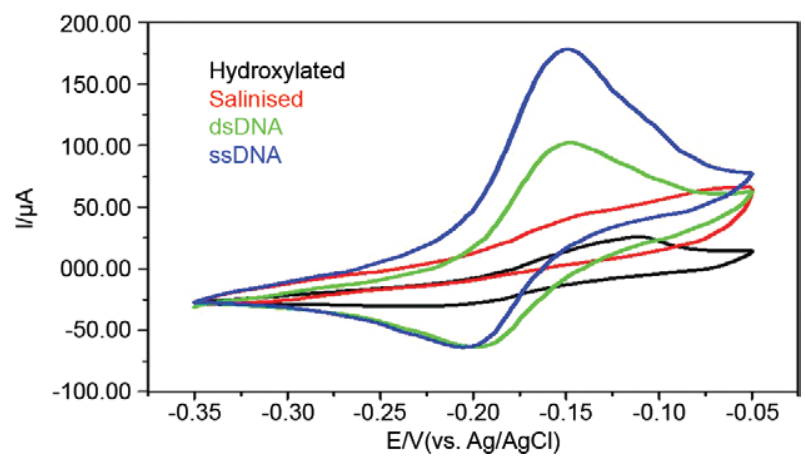

Fig. 4: cyclic voltammetry detection of single-stranded DNA (ssD$\mathrm{NA}$ ) and double-stranded DNA (dsDNA) in the presence of methylene blue. E/V: electrical potential/volts.

enabled amplification of ssDNA that was suitable for direct hybridisation in an electrochemical DNA biosensor. We have demonstrated the utilisation of ssDNA in probe immobilisation in the sequence-specific electrochemical detection of $W$. bancrofti. This method is reproducible and avoids many of the difficulties encountered in conventional methods of DNA detection that utilise carcinogenic substances such as $\mathrm{EtBr}$ and are time-consuming. This method has potential application in the xenomonitoring of filarial parasites.

\section{ACKNOWLEDGEMENTS}

To Dr P Jambulingam, Director, Vector Control Research Centre, for critical comments in preparing this paper, and to $\mathrm{R}$ Balasubramanian, for technical assistance.

\section{REFERENCES}

Farid HA, Hammad MM, Morsy ZS, Kamal IH, Weil GJ, Ramzy RMR 2001. Detection of Wuchereria bancrofti in mosquitoes by the polymerase chain reaction: a potentially useful tool for large scale control programmes. Trans Royal Soc Trop Med Hyg 95: 29-32.

Helmy H, Fischer P, Farid HA, Bradley MH, Ramzy RM 2004. Test strip detection of Wuchereria bancrofti amplified DNA in wild caught Culex pipiens and estimation of infection rate by a pool screen algorithm. Trop Med Int Health 9: 158-163.

Hoti SL, Patra KP, Vasuki V, Lizotte MW, Hariths VR, Ravi VR, Sushma S, Gunasekaran K, Ramaiah KD, Vanamail P, Williams SA, Mariappan T 2002. Evaluation of Ssp I PCR assay in the de- tection of Wuchereria bancrofti infection in field collected Culex quinquefasciatus and its application in the transmission studies of lymphatic filariasis. J Appl Entomol 126: 417-421.

Kaltenboeck B, Kousoulas KG 1996. Efficient PCR production of single-stranded DNA sequencing templates. Methods Mol Biol 65: 149-153.

Lai RY, Lagally ET, Lee SH, Soh HT, Plaxco KW, Heeger AJ 2006. Rapid, sequence-specific detection of unpurified PCR amplicons via a reusable, electrochemical sensor. Proc Natl Acad Sci USA 103: 4017-4021.

Li LL, Cai H, Lee TH, Barfood J, Hsing IM 2004. Electrochemical detection of PCR amplicon using electroconducive polymer modified electrode and multiple nanoparticle labels. Electroanalysis 16: 81-87.

Liang Z, Lao R, Wang J, Liu Y, Wang L, Huang Q, Song S, Li G, Fan C 2007. Solubilization of single-walled carbon nanotubes with single stranded DNA generated from asymmetric PCR. Int $\mathrm{J} \mathrm{Mol}$ Sci 8: 705-713.

Ottesen EA 2000. The global programme to eliminate lymphatic filariasis. Trop Med Int Health 5: 591-594.

Poddar SK 2000. Symmetric vs. asymmetric PCR and molecular beacon probe in the detection of a target gene of adenovirus. Mol Cell Probes 14: 25-32.

Rai V, Hapuarachi CH, Ng LC, Soh SH, Leo YS, Toh CS 2012. Ultrasensitive cDNA detection of dengue virus RNA using electrochemical nanoporous membrane-based biosensor. PLOS ONE 7: e42346.

Ramzy VK, Farid HA, Kamal IH, Ibrahim GH, Molrsi ZS, Faris R, Weil GJ, Williams SA 1997. A polymerase chain reaction basedassay for detection of Wuchereria bancrofti in human blood and Culex pipiens. Trans R Soc Trop Med Hyg 91: 156-160.

Vasuki V, Hoti SL, Sadanandane C, Jambulingam P 2003. A simple and rapid extraction method for the detection of Wuchereria bancrofti infection in vector mosquito, Culex quinquefasciatus by Ssp1 PCR assay. Acta Trop 86: 109-114.

Vasuki V, Subramanian S, Hoti SL, Jambulingam P 2012. Use of a simple DNA extraction method for high-throughput detection of filarial parasite Wuchereria bancrofti in the vector mosquitoes. Parasitol Res 111: 2479-2481.

WHO - World Health Organization 1997. Elimination of lymphatic filariasis as a public health problem - resolution of the executive board of the WHO. 50th World Health Assembly, May 6 1997, Geneva, p. 50.29.

Wooddell CI, Burgess RR 1996. Use of asymmetric PCR to generate long primers and single-stranded DNA for incorporating crosslinking analogs into specific sites in a DNA probe. Genome Res 6: 886-892. 